\title{
Monoclonal gammopathy of undetermined significance
}

\author{
Sébastien Anguille MD, Christian Bryant MD
}

\section{Monoclonal gammopathy of undeter- mined significance is a diagnosis of exclusion}

The condition, also known as benign monoclonal gammopathy, is characterized by the presence of a monoclonal immunoglobulin or light chain on immunofixation of the patient's serum or urine. ${ }^{1.2}$ Such a diagnosis can only be considered if underlying causes, most notably multiple myeloma, are excluded (Appendix 1, available at www.cmaj.ca/lookup /suppl/doi:10.1503/cmaj.122090/-/DC1). ${ }^{1}$ Because monoclonal immunoglobulins may be transient, this condition should only be diagnosed if they persist.

\section{All cases should be investigated}

Because this condition can evolve into multiple myeloma or, less frequently, other B-cell malignancies, investigation is required to exclude a malignant cause (Appendix 1). ${ }^{1}$ The condition is also associated with some nonmalignant diseases, including neuropathy, autoimmune disease and chronic infections with viruses such as HIV and hepatitis C. ${ }^{6}$ Most of these reported associations are coincidental and not causal. ${ }^{6}$

\section{References}

1. Kyle RA, Durie BG, Rajkumar SV, et al. Monoclonal gammopathy of undetermined significance (MGUS) and smoldering (asymptomatic) multiple myeloma: IMWG consensus perspectives risk factors for progression and guidelines for monitoring and management. Leukemia 2010;24:1121-7.

2. Dispenzieri A, Katzmann JA, Kyle RA, et al. Prevalence and risk of progression of light-chain monoclonal gammopathy of undetermined significance: a retrospective population-based cohort study. Lancet 2010;375:1721-8.

3. Greenberg AJ, Vachon CM, Rajkumar SV. Disparities in the prevalence, pathogenesis and progression of monoclonal gammopathy of undetermined

\section{Monoclonal gammopathy of undetermined significance is a common problem}

About $4.2 \%$ of people aged 50 years or older have this condition, and its prevalence increases with age - almost $10 \%$ of men older than 80 years are affected. ${ }^{2}$ It is more common in men than in women, and it occurs 2-3 times more frequently among black people than among white people. ${ }^{3}$ The available evidence suggests that this racial disparity is a function of genetic predisposition, ${ }^{3}$ although other risk factors, such as obesity ${ }^{3}$ and underlying HIV infection, ${ }^{4}$ may also contribute to the differences seen. There is no evidence to support population screening for the condition. Appendix 1 outlines indications for testing."

\section{Tools are available to assess the risk of evolution into malignant disease}

Almost all cases of multiple myeloma are preceded by monoclonal gammopathy of undetermined significance. ${ }^{5}$ Nevertheless, many patients have a very low risk of the condition undergoing malignant transformation, and they should be reassured. Proper risk stratification is important. ${ }^{5} \mathrm{~A}$ commonly used risk stratification tool is the Mayo Clinic model, in which 3 parameters are associated with increased risk of progression to multiple myeloma: a paraprotein concentration greater than 15 $\mathrm{g} / \mathrm{L}$, the presence of nonimmunoglobulin $\mathrm{G}$ paraprotein and an abnormal free lightchain ratio in the serum. ${ }^{5}$ The 20 -year absolute risk of multiple myeloma developing is only $5 \%$ in patients with no risk factors (low risk), but $56 \%$ in patients with all 3 risk factors (high risk). ${ }^{5}$

significance and multiple myeloma between blacks and whites. Leukemia 2012;26:609-14.

4. Konstantinopoulos PA, Pantanowitz L, Dezube BJ. Higher prevalence of monoclonal gammopathy of undetermined significance in African Americans than whites - the unknown role of underlying HIV infection. J Natl Med Assoc 2006;98:1860-1.

5. Berenson JR, Anderson KC, Audell RA, et al. Monoclonal gammopathy of undetermined significance: a consensus statement. Br J Haematol 2010;150:28-38.

6. Bida JP, Kyle RA, Therneau TM, et al. Disease associations with monoclonal gammopathy of undetermined significance: a population-based study of 17398 patients. Mayo Clin Proc 2009;84:685-93.

\section{No known treatment exists and follow-up should be individualized}

This condition requires no treatment, and no therapy is known to prevent its progression to multiple myeloma. $\mathrm{Pa}$ tients with monoclonal gammopathy of undertermined significance must be monitored for malignant transformation or toxicity related to the presence of paraprotein (e.g., renal impairment, neuropathy). Guidelines suggest follow-up and repeat serum electrophoresis 3-6 months after the initial diagnosis, with risk-adapted follow-up thereafter if the paraprotein concentration remains stable. Patients at intermediate or high risk should be seen every 3-6 months, whereas those at low risk require review every $2-3$ years.

\section{Competing interests: None declared.}

This article has been peer reviewed.

Affiliations: Division of Hematology (Anguille), Antwerp University Hospital, Antwerp, Belgium; and Dendritic Cell Biology and Therapeutics Group (Bryant), ANZAC Research Institute, Sydney, Australia

Correspondence to: Sébastien Anguille, sebastien .anguille@uza.be

CMAJ 2013. DOI:10.1503/cmaj.122090 